\title{
El cuidado como eje vertebrador de una nueva economía*
}

\author{
Cristina CARRASCO BENGOA \\ Universidad de Barcelona \\ Departamento de Teoría Económica \\ cristinacarrasco@ub.edu
}

Recibido: 10-07-2012

Aceptado: 17-09-2012

\section{RESUMEN}

En este artículo se plantean tres ideas que fundamentan, desde nuestra perspectiva, la economía feminista: la ruptura teórica con la economía oficial ampliando las fronteras más allá del mercado, el "descubrimiento" de los cuidados y el desafío político proponiendo una ruptura con el modelo vigente. Se pretende mostrar la potencialidad tanto teórica como política de la economía feminista, su capacidad para plantear un nuevo paradigma capaz de ofrecer una radiografía del funcionamiento socioeconómico mucho más ajustado a la realidad que el que ofrece habitualmente la economía.

Palabras clave: economía feminista, cuidados, condiciones de vida

\section{Care as the backbone of a new economy}

\begin{abstract}
This article presents three ideas that underpin, from our perspective, feminist economics: the theoretical break with the official economy to expand the boundaries beyond the market, the "discovery" of care and the political challenge by proposing a break with the current model. The aim is to show the theoretical and political potential of feminist economics, their ability to propose a new paradigm capable of providing a radiograph of social and economic founding much closer to reality than that provided by the economy.
\end{abstract}

Keywords: feminist economics, care, living conditions

REFERENCIA NORMALIZADA

Carrasco Bengoa, C. (2013). El cuidado como eje vertebrador de una nueva economía. Cuadernos de relaciones laborales, Vol. 31, núm. 1, p. 39-56.

SUMARIO: Introducción 1. Primera idea: la ruptura teórica 2. Segunda idea: el "descubrimiento" de los cuidados 3. Tercera idea: el desafío político 4. Epílogo: crisis y condiciones de vida 5. Bibliografía.

* Este artículo puede considerarse una reflexión colectiva, ya que incorpora ideas que hemos ido acuñando y desarrollando entre diversas economistas feministas. Por sus importantes aportaciones, quisiera nombrar de forma explícita a Antonella Picchio y a Amaia Pérez Orozco. 


\section{Introducción}

Aunque con antecedentes más tempranos ${ }^{1}$, el trabajo doméstico y de cuidados ${ }^{2}$ se constituye hace cuatro décadas en un tema central de debate del movimiento feminista $\mathrm{y}$, posteriormente, de la economía feminista. La reflexión sobre este trabajo se inicia a finales de los años sesenta del siglo XX con el llamado "Debate sobre el trabajo doméstico" y a medida que se van discutiendo y visibilizando algunas de sus características o funciones, se va utilizando una terminología diferente que permitiese incluir y nombrar esos nuevos elementos "descubiertos". En este itinerario aún no acabado, ninguno de los términos acuñados ha sido totalmente satisfactorio y eso no es ni por casualidad ni porque el pensamiento feminista manifieste alguna "incapacidad conceptual". Más bien es una clara señal de que estamos frente a un proceso muy complejo; con elementos conceptuales, teóricos, sociales y políticos; adaptable históricamente; y absolutamente necesario para la reproducción social y el bienestar de las personas. Proceso que, curiosamente, teniendo en cuenta su importancia, lleva escaso tiempo siendo preocupación como ámbito de estudio: cuatro décadas son casi nada comparado con los casi tres siglos de estudio de la economía o los seis de otras áreas de conocimiento. Más aún, cuatro décadas donde ha sido el pensamiento feminista casi en exclusiva quien se ha preocupado del tema intentando continuamente vencer la fuerte oposición de las disciplinas sociales oficiales que se resisten a la integración de este trabajo en sus marcos analíticos y de las organizaciones -digamos emancipadoras-, sociales y políticas, que se resisten a incluirlo en sus hojas de ruta. De hecho, toda esta resistencia social, académica y política, es bastante comprensible, ya que no estamos hablando de ninguna tontería. No se trata de agregar un capítulo más a los manuales al uso o una reivindicación más a los programas electorales. Se trata nada más ni nada menos que de un cambio de paradigma, de otra manera de mirar el mundo; donde casi todo lo aprendido hasta ahora se transforma, desde el enfoque hasta el objetivo social, pasando por conceptos, políticas, estadísticas, etc.

El recorrido conceptual se inició con el debate sobre la naturaleza del trabajo doméstico y su relación con el modo de producción capitalista. Los y las participantes en el debate eran de filiación feminista, marxista o ambas. La tradición marxista de muchos de ellos y ellas quedó reflejada en el grueso de los temas abordados, aunque particularmente en la tesis -sostenida mayoritariamente- de que el trabajo

${ }^{1}$ Se acostumbra a señalar a Margaret Reid (1934) como la primera autora que nombra y analiza la producción doméstica desde la economía. Pero también hay que recordar que ya en el siglo XIX había mujeres que discutían el tema del trabajo doméstico no asalariado; ver por ejemplo, Heather-Bigg (1894) y Perkin Gilman (1898).

2 En este artículo, al trabajo realizado desde los hogares sin remuneración, lo nombraremos como trabajo doméstico y de cuidados, aunque precisamente la terminología con que se designa dicho trabajo es un problema no resuelto que se comentará a continuación. 
doméstico respondía a una necesidad del capitalismo, fundamentalmente, como participante en la reproducción de la fuerza de trabajo. Se manifestaba así el carácter económico/mercantil del debate al centrarse sólo en la reproducción de la fuerza de trabajo y no en la satisfacción de las necesidades de toda la población.

Más adelante, el término de trabajo doméstico se fue abandonando para pasar a llamarse trabajo reproductivo, en oposición al trabajo mercantil conocido como el productivo. Sin embargo, esta nueva denominación también presentaba problemas. Por una parte, la actividad con definición y valoración propia continuaba siendo la producción mercantil y la otra, la re-producción, sólo podía existir en la medida que se reflejaba en la anterior. La noción de "lo productivo" se continuaba reservando para el mercado. Una segunda limitación de esta denominación era que mantenía una visión dicotómica (productivo-reproductivo) que en el feminismo, desde la obra de Carole Pateman (1988), estaba muy criticada. Y, un tercer problema, se presentaba fundamentalmente con la disciplina económica. En economía se conoce como enfoque reproductivo a aquella perspectiva de análisis que tiene en cuenta no solo los procesos productivos, sino también las condiciones de reproducción de dichos procesos. Estos enfoques, a nivel teórico, comienzan con David Ricardo, continúan con Carlos Marx y actualmente han sido desarrollados por la llamada escuela sraffiana.

Lentamente, durante este proceso de conceptualización, desde la academia y el movimiento de mujeres se comienza a recuperar y a dar valor a la experiencia de las mujeres, a reconocer los valores propios del trabajo doméstico como valores humanos fundamentales. Valores, que hasta entonces habían permanecido ocultos bajo el peso del mercado en una sociedad patriarcal capitalista. Desde esta nueva perspectiva, las mujeres no eran ya personas secundarias y dependientes sino personas activas, actoras de su propia historia, creadoras de culturas y valores del trabajo distintos a los del modelo masculino ${ }^{3}$.

El término "trabajo familiar doméstico" fue acuñado para incorporar el trabajo de mediación -realizado fuera del espacio familiar- entre las nuevas necesidades familiares y los servicios ofrecidos por el estado de bienestar (Balbo 1980). E, incluso, de forma genérica, se utilizó el término de "trabajo no remunerado", término poco preciso ya que, por una parte, incluye cualquier trabajo que no tenga contrapartida monetaria, como el trabajo voluntario o el trabajo familiar, realizado sin remuneración por miembros de una familia en la empresa familiar; y, por otra, hay un debate pendiente sobre la remuneración del trabajo no asalariado realizado desde los hogares: ¿está remunerado? ¿cómo? ¿por quién? ¿la propia persona que lo realiza?

En este contexto, el análisis cada vez más profundo y minucioso en relación al contenido de las actividades desarrolladas en el hogar, llevó -de manera bastante natural- a plantear los aspectos subjetivos de las necesidades y el importante papel que jugaba el "trabajo de cuidados". De esta manera, el "cuidado", con todas las

\footnotetext{
${ }^{3}$ Estas ideas se desarrollan en la Introducción de Amoroso et al. 2003.
} 
connotaciones más subjetivas, comenzó a emerger como un aspecto central del trabajo doméstico. La identificación de estos aspectos emocionales y relacionales del cuidado - que tienen que ver directamente con la calidad de vida de las personas y el bienestar humano- planteó cada vez más la necesidad de valorar esta actividad por sí misma, de reconocerla como el trabajo fundamental para que la vida continúe. En consecuencia, era necesario un cambio de paradigma: el objetivo social debieran ser las personas y no el capital, con lo cual, era necesario tomar como eje social el trabajo de cuidados y no en el trabajo y la producción de mercado (Amoroso et al. 2003, Bosch et al. 2005, Pérez Orozco 2006, 2007a, Picchio 2001, 2009).

Este camino inacabado del estudio del trabajo de cuidados incluye aspectos teóricos, conceptuales y políticos, que aún requieren mucho estudio y debate, pero que ya pueden ser presentados como tres ideas que fundamentan la economía feminista ${ }^{4}$. Pero antes quisiera hacer una pequeña reflexión sobre cómo el conocimiento aprendido -y continuamente trasmitido- puede ofrecer perspectivas que se nos presentan como las únicas posibles e impiden realizar análisis que no sean bajo el paradigma dominante. Utilizaré un ejemplo. Existen unos mapas del mundo relativamente conocidos, elaborados originalmente en Australia. Están construidos girando y desplazando los habituales, con lo cual Australia sustituye a Europa como región central. La versión de las personas que los han elaborado es que están cansadas de los mapas construidos por los colonizadores europeos. Ahora bien, si dichos mapas se muestran sin más información a un grupo de personas, lo más probable es que más de alguien diga inmediatamente "está al revés". Frente a esto una se pregunta, ¿cómo un mapa puede estar "al revés" si lo que representa es un globo que se mueve por un espacio sin referentes, al menos, desde nuestra perspectiva? La visión que nos han enseñado siempre nos parece tan única que la primera reacción es decir que está al revés. Y si intentamos situar cualquier país o ciudad tenemos una dificultad añadida; con el mapa habitual nos sabemos situar porque lo tenemos interiorizado, con el nuevo nos perdemos.

Con la economía sucede algo semejante al mapa. Hemos aprendido una visión que se nos ha presentado como única y, sin embargo, es parcial y distorsionadora. La economía feminista cambia la perspectiva, flexibilizando y ampliando la mirada para incorporar la experiencia de las mujeres; lo que permite reformular conceptos centrales del análisis económico, desarrollar nuevos marcos analíticos y elaborar políticas públicas que den respuesta a la realidad de mujeres y hombres.

\section{Primera idea: la ruptura teórica}

Desde Adam Smith y hasta el presente, todos los estudios económicos, ya sean modelos teóricos o estudios de economía aplicada tienen lugar dentro de las fronte-

${ }^{4}$ La economía feminista, afortunadamente, no es un pensamiento único, por lo cual muchas economistas feministas pueden no estar de acuerdo con este planteamiento. 
ras del mercado. Con diferencias en sensibilidades o matices en el reconocimiento de la existencia del trabajo no asalariado realizado desde los hogares, las distintas escuelas de pensamiento económico se han mantenido en los márgenes de la economía monetizada. Los enfoques más heterodoxos denuncian las desigualdades resultantes de la economía capitalista de mercado: la pobreza, las desigualdades de renta, la inexistencia de estados del bienestar, etc., pero sin integrar el trabajo doméstico y de cuidados en sus análisis ni considerar las enormes desigualdades que se generan entre hombres y mujeres al tener estas últimas asignado dicho trabajo. Así, el trabajo mercantil es el único considerado como tal, convirtiéndose el término trabajo en sinónimo de empleo.

En consecuencia, para la economía, las empresas contratan tiempo de trabajo (empleo) que remuneran con un salario, dicho salario permite a los hogares adquirir todos los bienes y servicios necesarios para la subsistencia de las personas del hogar, reproduciéndose de esta manera la fuerza de trabajo que vuelve a ser contratada por las empresas. En ningún momento se considera la necesidad de realizar trabajo doméstico y de cuidados. De hecho, cuando los pensadores clásicos hablaban de salario de subsistencia, consideraban un salario que permitiese adquirir en el mercado los bienes necesarios para la subsistencia de la familia. Y cuando Carlos Marx acuña el término de plusvalía, está siendo heredero de la misma tradición.

Desde la economía feminista se critica esta mirada estrecha que la economía sostiene sobre el trabajo y se ofrece una perspectiva mucho más realista que amplía el circuito del trabajo integrando lo que puede designarse como la economía del cuidado. Si se comienza con la misma idea anterior, que las empresas adquieren trabajo a cambio de un salario, cuando dicho salario llega a los hogares permite adquirir una serie de bienes y servicios, pero estos no son en ningún caso suficientes para reproducir a la población y, en particular, a la fuerza de trabajo. Es absolutamente necesario realizar otro trabajo que en parte va destinado a transformar los bienes adquiridos en el mercado (como, por ejemplo, la comida) y, en parte, a realizar servicios: de limpieza, de gestiones, de cuidados, etc. ${ }^{5}$. Ahora bien, la separación entre los dos tipos de trabajo no es rígida, más bien es porosa y cambiante, dependiendo del desarrollo tecnológico y de los niveles de renta, tanto de las sociedades en general como de los hogares en particular. A lo largo de la historia, en las sociedades con mayor desarrollo industrial, producciones que tradicionalmente se habían realizado en los hogares -como la confección de la ropa- cada vez más son

${ }^{5}$ El esquema que aquí se presenta es un esquema simple que no pretende representar todos los trabajos y dineros necesarios para la reproducción. El objetivo es mostrar cómo cambia la perspectiva de análisis cuando se incorpora el trabajo doméstico y de cuidados en el análisis. Es sabido que los hogares, además de salarios y trabajo doméstico y de cuidados, pueden recibir aportaciones del sector público en términos de servicios o transferencias. Aunque es desde los propios hogares desde donde se gestiona y organiza todo el mantenimiento y cuidado de las personas. 
desplazadas al mercado. Pero en épocas de crisis como la actual, dichas producciones pueden regresar a los hogares. Por ejemplo, los niños y niñas ya no se quedan a comer en el colegio si el nivel de renta del hogar ha disminuido, o se comienza a retirar a las personas mayores de las residencias, procesos que hoy están teniendo lugar en España. Por otra parte, hay situaciones en que las personas realizan dos tipos de trabajo simultáneamente, sin que se pueda determinar la frontera entre ellos. Es el caso, por ejemplo, en sociedades pobres, donde muchas mujeres que trabajan en venta ambulante van acompañadas de sus hijos e hijas menores; entonces, ¿se trata de autoempleo o trabajo de cuidados? ¿o de ambos? O, en sociedades no necesariamente pobres, cuando el trabajo remunerado se realiza desde el hogar (traductoras, informáticas, etc.), muchas mujeres están simultáneamente gestionando tareas del hogar y realizando el trabajo remunerado. Finalmente, un tercer elemento que interviene para que las fronteras entre la economía mercantil y la economía del cuidado sean difusas, es la existencia de otro tipo de trabajo -relevante en nuestras sociedades- que no está monetizado (no es empleo) pero no se desarrolla desde los hogares ni se dirige a sus miembros, es el trabajo de participación ciudadana, comúnmente llamado, trabajo voluntario.

Los bienes y servicios producidos desde el ámbito doméstico, por una parte, incrementan la renta nacional, cuestión que la economía nunca ha considerado en el Producto Interior Bruto. Y con esta afirmación solo se pretende destacar lo mal medido que está el PIB y no insistir en la valoración monetaria del trabajo doméstico y de cuidados, tema que continúa en debate. Pero, por otra, el trabajo realizado desde los hogares proporciona aspectos emocionales, de socialización, de cuidado en la salud, en la vejez, etc., muchos de ellos imposibles de ser adquiridos en el mercado. Lo cual implica algo que va mucho más allá de la mera existencia biológica: la reproducción como personas humanas y sociables.

Así, el trabajo y la gestión realizada desde los hogares reproduce y cuida a toda la población y, en particular, reproduce la fuerza de trabajo diaria y generacional necesaria para la subsistencia del sistema de producción capitalista. Dicho sistema económico no tiene capacidad de reproducir la fuerza de trabajo bajo sus propias relaciones de producción. Si eso fuese así, significaría que solo con el salario toda la población tendría la posibilidad de reproducirse sin necesidad de realizar ningún otro tipo de trabajo. Incluso sin tener en cuenta los aspectos emocionales difícilmente mercantilizables, la pregunta sería: ¿qué salario deberíamos ganar todos y todas para que trabajando digamos 40 horas semanales en el mercado pudiésemos adquirir todos los bienes y servicios en el mercado: compras, elaboración de las comidas, limpieza, gestiones varias, lavado y planchado de la ropa, cuidado de las criaturas y de las personas mayores, etc.? El sistema capitalista no podría subsistir sin el trabajo doméstico y de cuidados, depende de él para el mantenimiento de la población y la reproducción de la necesaria fuerza de trabajo. Lo cual significa que desde los hogares estamos entregando a las empresas una fuerza de trabajo por 
debajo de su coste real, ya que en el coste de reproducción de dicha fuerza de trabajo no se tiene en consideración las energías y el tiempo dedicado a reproducirla que viene desde los hogares ${ }^{6}$. Esta es una razón importante para mantener oculto, no ya el trabajo doméstico, sino la fuerte relación que mantiene con el sistema capitalista; relación que le permite a este último poder reproducirse.

Por tanto, se puede fácilmente concluir que parte del beneficio de la empresa privada proviene de la utilización de la unidad doméstica - tratada como caja negray no solo de la explotación en el puesto de trabajo, como pensaba Carlos Marx, entre otros. El capitalismo se construye así sobre una inmensa masa de trabajo no asalariado ni basado en relaciones contractuales, que hace posible la acumulación de capital (Federici 2010a). Ahora bien, en un sistema patriarcal, la apertura de dicha caja negra, muestra que el trabajo doméstico y de cuidados continúa recayendo fundamentalmente sobre las mujeres, como muestran los datos de diferentes encuestas ${ }^{7}$. Y también es importante recordar que la dificultad del trabajo doméstico y de cuidados realizado básicamente por las mujeres, no es tanto el número de horas de trabajo -que también- sino la lucha por dar prioridad a las necesidades de las personas frente a las restricciones y exigencias del beneficio. Lo cual se traduce en un enorme conflicto de organización de los tiempos de la vida cotidiana.

Por otra parte, en nuestras sociedades actuales, la subsistencia de las personas no está asegurada solo con el trabajo realizado desde los hogares. No somos autosuficientes en la producción de los bienes y servicios y, por tanto, requerimos de dinero para adquirir una serie de productos en el mercado. Por consiguiente, lo que es

${ }^{6}$ Lo cual significa que al devaluar el trabajo doméstico y de cuidados, se ha devaluado también su producto, la fuerza de trabajo.

${ }^{7}$ La encuesta de referencia para este tipo de información es la Encuesta de Empleo del Tiempo (EET) (INE), que en España se ha realizado dos veces para todo el territorio, en $2002 / 2003$ y en $2009 / 2010$. También hay otras encuestas que incorporan preguntas sobre realización de trabajo doméstico y de cuidados, como la Encuesta Sociodemográfica de Catalunya 2007 (IDESCAT) o la Encuesta Nacional de Salud de España 2006 (INE).

Según información de la EET 2009/2010, en España, mujeres y hombres dedican respectivamente como media social 4 horas 7 minutos y 1 hora 54 minutos diarios respectivamente al trabajo doméstico y de cuidados. $\mathrm{Y}$ aunque estas cantidades son algo inferior que en 2002/2003 para las mujeres y algo superior para los hombres, las diferencias entre ambos siguen siendo muy marcadas. Pero el tiempo total de trabajo de unas y otros sigue manteniendo una hora de diferencia a favor de las mujeres. Por otra parte, el tiempo medio social dedicado diariamente a trabajo de mercado considerando toda la población de 10 y más años es de 2 horas 27 minutos y el dedicado a trabajo doméstico y de cuidados es de 2 horas 44 minutos. Esta última información permite observar que para vivir en las condiciones que está viviendo la sociedad española, por persona y día se está dedicando más tiempo al trabajo realizado en los hogares que al trabajo de mercado (17 minutos diarios de diferencia). Todo ello, teniendo en cuenta que el trabajo de cuidados no queda bien recogido y, por tanto, su valor real es muy superior al que ofrecen las encuestas. 
importante de destacar es que existe una dependencia mutua entre ambos tipos de trabajos, una relación dinámica entre el proceso de producción y reproducción de mercancías y el proceso de reproducción de la población y, en particular, de la fuerza de trabajo.

El debate pendiente -iniciado pero nunca acabado- es si el trabajo doméstico y de cuidados debe orientarse fundamentalmente a reproducir fuerza de trabajo o a socializar personas que puedan realizar una "vida que valga la pena ser vivida". En algunos aspectos ambos objetivos no son contradictorios, pero en otros muchos sí lo son. Por ejemplo, el tipo de valores que requiere uno u otro no son los mismos: individualismo frente a solidaridad; beneficio individual frente a beneficio social, reconocimiento de un tipo de trabajo y no del otro, tipos de estudios necesarios de realizar porque son los valorados en el mercado, etc. Debate muy complejo en razón de las restricciones y presiones de las sociedades en que vivimos, pero necesario si se quiere optar por sociedades alternativas.

El enfoque amplio propuesto desde la economía feminista también ha permitido analizar con mayor claridad y rigor la pobreza específica de las mujeres. La responsabilidad de las mujeres en el trabajo doméstico y de cuidados, que les impide trabajar en las mismas condiciones que los hombres en el mercado, resulta en menores salarios y menores pensiones en la vejez. Pero el modelo masculino de trabajo en el mercado no es generalizable, ya que implica libertad de tiempos y acciones, lo cual no es compatible - ni conciliable- con responsabilidad sobre el cuidado de las personas, sobre todo con aquellas que no tienen capacidad de realizar las acciones habituales de la vida cotidiana. En consecuencia, una posible "igualdad" solo podrá realizarse con un cambio de modelo, pero no intentando integrar a las mujeres al modelo masculino de empleo.

El envejecimiento demográfico junto a la cada vez mayor participación laboral de las mujeres ha llevado a una situación crítica, puesto que la oferta de trabajo de las mujeres no era infinita, como parece que se presuponía. Una situación crítica en relación al cuidado de las personas, básicamente, de las personas mayores muy dependientes que requieren presencia constante de otra persona adulta. Dicha situación, unida a la incapacidad o no disposición para pensar en un cambio de modelo de trabajo ha llevado a la denominada crisis de los cuidados. "Crisis" que las clases medias y altas han resuelto de manera individual contratando mujeres pobres de países más pobres. El cuidado, aunque nos atañe a todas las mujeres $-\mathrm{y}$ lo realizamos en redes de abuelas, madres, hijas, vecinas, hermanas-, existen grandes diferencias, tanto en cuanto a tiempo de dedicación como a condiciones de realización, según determinadas características como clase social, etnia, raza, etc. Un aspecto derivado de lo anterior son las cadenas mundiales de cuidados: mujeres pobres de países más pobres que emigran a cuidar a personas de países más ricos, los cuales 
no han sabido, querido o podido resolver sus propios problemas de subsistencia social $^{8}$.

En definitiva, la economía feminista al traspasar las fronteras estrechas de la economía y observar la realidad más allá del paradigma de mercado, ha permitido incorporar nuevos análisis y, particularmente, ha redefinido el concepto de trabajo. Pero también ofrece una herramienta para superar la manera en que el capital ha dividido a hombres y mujeres en los distintos trabajos. Esa división recreada y reproducida continuamente es una de las principales debilidades que impide construir una sociedad más equitativa (Federici 2010b) ${ }^{9}$.

\section{Segunda idea: el "descubrimiento" de los cuidados}

La profundización de los estudios sobre el trabajo familiar doméstico y su importancia en la reproducción y cuidado de las personas llevó a conceptualizar el trabajo de cuidados. Los primeros estudios nacieron de la mano de la sociología, aunque hay algunas economistas feministas que escriben sobre el tema a principios de los años noventa del siglo XX (Folbre 1995, Himmelweit 1995).

A diferencia del trabajo de mercado cuyo objetivo final es la producción de un bien o servicio que permita obtener un beneficio económico (si se trata de una empresa privada), y cuyo fin directo para la persona que lo realiza es la obtención de dinero; el trabajo realizado desde los hogares tiene como fin al cuidado de las personas a lo largo del ciclo vital, con las dependencias específicas que implica cada etapa de la vida y en sus distintas dimensiones: cuidados directos afectivos y emocionales, cuidado del entorno, cuidado de las relaciones, cuidados en la salud y en las dependencias específicas, producción de determinados bienes y servicios,

\footnotetext{
${ }^{8}$ No nos referimos a un problema de explotación de mujeres por mujeres como se acostumbra a decir. Una afirmación de este tipo estaría suponiendo que el trabajo doméstico y de cuidados es tema de mujeres y, de ahí, que a ella le corresponde resolverlo. Hay que entender que los hombres también son cómplices de dicha situación ya que la mujer contratada también realizará la parte de trabajo que le correspondería al hombre.

La temática sobre las cadenas globales de cuidados está ampliamente tratada en un estudio dirigido por Amaia Pérez Orozco (2007b).

${ }^{9}$ En su excelente libro, Federici (2010a) plantea la necesidad del capitalismo -tanto en su acumulación originaria como en sus sucesivos estadios de desarrollo- de separar los trabajos (entre aquellos dirigidos a la producción de mercado y aquellos dirigidos a satisfacer las necesidades de las personas del hogar) y asignarlos a hombres y mujeres respectivamente, siendo los segundos absolutamente necesarios para el funcionamiento del sistema y quedando subordinados a los primeros. Esta tesis nos tendría que hacer pensar sobre su posible vigencia en la crisis actual, en que la reprivatización de la reproducción social no es solo una necesidad por la "austeridad" sino una estrategia consciente y necesaria de un nuevo proceso de acumulación capitalista.
} 
gestiones diversas para el buen funcionamiento del hogar, etc. Todo ello hace del hogar el nicho básico donde las personas sentimos y vivimos los primeros lazos afectivos, donde aprendemos a relacionarnos y emocionarnos, donde crecemos y nos desarrollamos, donde nos socializamos y adquirimos los primeros valores, donde comemos, descansamos y nos reponemos cada día, donde realizamos una parte importante de nuestra vida cotidiana, la que tiene que ver con intereses y humanidades que están más allá del funcionamiento del mercado. Todo lo que en conjunto se ha denominado la tarea civilizadora de las mujeres, que no es otra cosa que el haber apostado por la vida, incluso en circunstancias históricas muy difíciles y complejas. A este respecto se puede citar a Boulding, que en 1972 afirmaba, "La supervivencia de la raza humana ha dependido primero de la explotación de las mujeres, sin la cual hace mucho tiempo que hubiese desaparecido" (Boulding 1972: $115)^{10}$.

Probablemente, las características señaladas del cuidado que tienen que ver con el cuidado de los cuerpos, los afectos y la vida, han sido un hilo conductor a lo largo de la historia, ya que han permitido que las sociedades no solo se reprodujeran en términos biológicos, sino que continuaran desarrollando vínculos y relaciones que hacen la vida más humana. Desde las comunidades más primitivas hasta nuestras sociedades actuales, los trabajos de cuidados -directos e indirectos- han ocupado una parte importante de nuestro tiempo -y nuestra energía-, mayor que el tiempo que dedicamos al mercado, como indican las correspondientes estadísticas ${ }^{11}$. Y, sin embargo, continúan siendo invisibles para la economía y la política y la mayoría de los economistas no los considera objeto de estudio por no estar orientados al mercado.

Por la relevancia en el mantenimiento de los cuerpos, el bienestar de las personas, la creación y recreación de la vida, la llamada economía del cuidado o sencillamente el cuidado, es reconocida desde la economía feminista como el ámbito fundamental al cual debieran dirigirse los objetivos sociales y económicos. Si el trabajo de cuidados tiene como objetivo el cuidado de la vida en sus aspectos tanto físicos como emocionales, dicha actividad es la que debiera servir de referente y no la actividad desarrollada en el mercado. Este desplazamiento del eje y objetivo social y económico representa un profundo cambio de paradigma, sobre el cual volveremos en el siguiente apartado.

Ahora bien, en la producción de mercado -a excepción de algunos servicios, como los servicios de proximidad- la persona que produce el bien está alejada de

${ }^{10}$ También en el sentido de Boulding (1976) se puede sostener que las sociedades tienen una "deuda social" para con las mujeres, ya que sin la donación de trabajo y energías que históricamente han realizado las mujeres hacia los hombres y hacia la sociedad, la vida de los hombres y de las nuevas generaciones sería insostenible, al menos, en las condiciones sociales actuales.

${ }^{11}$ Anteriormente ya se comentó que actualmente por persona de 10 y más años se están dedicando en España 2 horas 27 minutos a trabajo de mercado y de 2 horas 44 minutos a trabajo doméstico y de cuidados (EET 2009/2010). 
aquella que lo va a adquirir y consumir, su actividad acaba en la producción del bien, y no sabe ni interesa a quienes va dirigido. Y el fin básico es el dinero recibido o el beneficio que pueda reportar el bien o servicio producido; sin negar que existen trabajos muy satisfactorios de realizar. En cambio, el trabajo desarrollado desde el ámbito más cercano del hogar es inseparable de la persona que lo realiza. El objetivo, además del producto acabado, es la relación entre las personas que pueden identificarse como cuidadoras y cuidadas en una etapa del ciclo vital o pueden alternar roles en otro momento de la vida. Así, el objetivo no es solo el bien o servicio a producir, sino también y de forma relevante, la relación con la otra u otras personas que se establece durante el proceso, el vínculo creado en la relación. Además de la necesidad que se va a satisfacer, existe una motivación en la realización del cuidado que tiene que ver con los afectos o con un sentido de la responsabilidad para con los demás, sin esperar una remuneración monetaria. Un riesgo de esta visión es la construcción de una identidad femenina basada en el cuidado y la maternidad, lo que se ha denominado la "mística del cuidado", entendida como autosacrificio deseado por las mujeres. La idealización del cuidado realizado bajo supuestos de amor y altruismo ha sido enarbolada tradicionalmente por el patriarcado religioso. De esta manera es fácil negar la gran dureza que muchas veces representa el cuidado, que no siempre el realizarlo es un deseo sino una obligación moral socialmente construida que presiona a las mujeres, y que en muchas situaciones no cumple los requisitos de amor que se le supone.

En la tradicional división por sexo del trabajo que asigna el trabajo más ligado al hogar a las mujeres y el trabajo que se retribuye con dinero a los hombres, la experiencia femenina del trabajo - ligada fuertemente al trabajo de cuidados- ha estado permanentemente orientada hacia la relación, en cambio, la experiencia masculina se ha orientado hacia el resultado (Bosch et al. 2005). En muchos aspectos del trabajo de cuidados hay un plus de afectividad no sustituible. Aunque, lamentablemente, el carácter relacional, afectivo y emocional del trabajo de cuidados solo se hace evidente cuando, por algún motivo, este trabajo pierde su vertiente afectiva. La constatación de la importancia del trabajo de cuidado para el bienestar, el desarrollo de las personas y las relaciones humanas nos lleva a pensar -en contra de lo que es la valoración social- que es precisamente el trabajo de mercado el que ha quedado desvirtuado y devaluado al eliminar la relación humana que intrínsecamente incorporaba la experiencia femenina del trabajo.

Esta monetarización del trabajo distorsiona su reconocimiento como actividad cuya finalidad es la calidad de vida humana; convirtiéndolo en una actividad que sólo proporciona dinero para disponer de capacidad de consumo. Bajo esta nueva mirada que aporta la experiencia femenina del trabajo, el empleo no es más que lo que podríamos llamar un "trabajo empobrecido" (Bosch et al. 2005: 333).

Trabajo empobrecido porque ha sido despojado de la relación, de la parte más humana del trabajo. De aquí la importancia de recuperar el valor y reconocimiento 
de lo que ha sido tradicionalmente la actividad fundamental de las sociedades, aquella que les permite su subsistencia y reproducción en condiciones de humanidad.

Finalmente, quisiera destacar dos aspectos relevantes en torno al cuidado. El primero de ellos tiene que ver con las desigualdades entre las propias mujeres. Aunque la asignación del trabajo doméstico al sexo femenino sea universal y atraviesa todas las clases sociales, las condiciones en que se realiza el trabajo y la cantidad de trabajo a realizar $-\mathrm{y}$, por tanto, la situación de las mujeres- es muy distinta dependiendo de la clase social o nivel de renta de cada hogar. El segundo aspecto, guarda relación con los objetivos del cuidado. Aunque el objetivo genérico sea el cuidar a todas las personas, dar bienestar a la población; también existe un objetivo más específico propio de una sociedad patriarcal y es liberar de tiempo y responsabilidades familiares a los hombres adultos para que salgan a trabajar libres de restricciones al mercado, al espacio que tiene valor y reconocimiento social en una sociedad capitalista. El funcionamiento del mercado y el Estado presupone ese soporte vital -que realizan básicamente las mujeres a través de una red de interdependencias- sin el cual no dispondrían de fuerza de trabajo socializada y emocionalmente estructurada y segura (Carrasco et al. 2011).

\section{Tercera idea: el desafío político}

Esta nueva mirada de la economía desde la experiencia femenina no es solo como se puede deducir fácilmente- una nueva conceptualización del trabajo; sino un cambio de paradigma que transforma la economía y la política. Transforma la economía porque exige una mirada inclusiva que tenga en consideración las distintas actividades necesarias de realizar para posibilitar la reproducción social en condiciones aceptables para toda la población. Una teoría que no tenga en cuenta las necesidades de reproducción de los miembros que constituyen la comunidad no estará en condiciones de dar respuesta a medio o largo plazo a los problemas de supervivencia y bienestar que necesariamente se van a presentar. Con una mirada estrecha de la economía que solo considera el mercado, se implementarán políticas públicas, sociales y de empleo, que pueden ser erróneas en el sentido de no cumplir sus objetivos, ya que se elaboran sin tener en cuenta los efectos producidos en la "cara oculta" de la economía. En el lenguaje al uso, serían "externalidades".

Las personas que venimos de alguna tradición de izquierda o emancipatoria, habíamos estudiado, leído y escuchado que la contradicción social fundamental tenía lugar entre beneficios y salarios. No se puede negar la importancia de la lucha histórica entre los dueños del capital y los o las trabajadores/as. Pero al ampliar la mirada, se observa que existen otras tensiones más allá de la que responde a los mecanismos capitalistas, en nuestro caso, nos interesa nombrar las contradicciones que genera el patriarcado, que se dirimen en todos los campos sociales, aunque con 
más fuerza en el ámbito del hogar, donde las mujeres se encuentran en situaciones de mayor fragilidad ${ }^{12}$. Sin embargo, seguramente lo más relevante de mantener una perspectiva más realista es la posibilidad de definir una contradicción social global: la que se establece entre capital y vida. El salario es un factor que afecta a las condiciones de vida, pero no es el único, tan importantes son el trabajo de cuidados realizado desde los hogares como los posibles servicios y transferencias que pueda ofrecer el sector público.

El objetivo del espacio de producción mercantil capitalista es la obtención de beneficio, por el contrario, el objetivo del espacio del cuidado es el bienestar de las personas. Dos objetivos contrarios, absolutamente irreconciliables. La opción es optar por uno de ellos y poner a su servicio el otro. Nuestras sociedades, o quienes tienen el poder en nuestras sociedades, han apostado por el capital y así, las personas han quedado al servicio de la acumulación de capital por parte de unos pocos. La transformación y ruptura política con el modelo oficial que plantea la economía feminista es desplazar el objetivo del beneficio hacia el objetivo de la vida, hacer una apuesta por las personas y no por el capital. Cuestionar el modelo vigente representa pensar un mundo común para mujeres y hombres más allá del discurso dominante; y más allá de la simple idea de igualdad. La economía feminista está proponiendo otra manera de mirar el mundo, otra forma de relación con el mundo, donde la economía se piense y realice para las personas. Esta propuesta representa un cambio radical, ya que exige: una reorganización de los tiempos y los trabajos (mercantil y de cuidados), cambios en la vida cotidiana, una nueva estructura de consumo y de producción y, por supuesto, un cambio de valores.

Se podrá decir que esta idea también es compartida por otros grupos o movimientos sociales, sin embargo, la diferencia está en lo que dichos grupos no contemplan en su definición de "buenas condiciones de vida" o "buen vivir". Los colectivos que sostienen esta idea generalmente mantienen una perspectiva multidimensional del bienestar en la línea elaborada por Amartya Sen, donde se incluyen aspectos como salud, educación, alimentación, etc., pero con una visión que nos parece estrecha porque mantiene la mirada masculina que solo tiene en cuenta el mundo más allá de los hogares. Mirada masculina que se traduce fundamentalmente en dos aspectos. En primer lugar, todos los ámbitos -laboral, de salud, vivienda, etc.- pueden tener una mirada masculina ${ }^{13}$; $y$, en segundo lugar, los colectivos que se manifiestan a favor del buen vivir nunca suelen incluir en dicho concepto el hecho del cuidado, el hecho de que un elemento central y necesario en el bienestar a lo largo de todo el ciclo vital es el estar cuidado/a. Esta es una especificidad lamentablemente y exclusivamente del discurso feminista.

12 También existe una tensión planteada desde la ecología entre nuestra forma de producir y consumir y las condiciones de sostenibilidad del planeta. Pero, este tema desborda el objetivo de este artículo.

${ }^{13}$ Un análisis de este aspecto se puede ver en Carrasco et al. 2006. 
En relación a la reflexión sobre qué significa buenas condiciones de vida o los diversos nombres que ha tomado este objetivo, contamos con algunos debates ya iniciados, aunque todavía es necesario continuarlos. El significado de "buen vivir" o "vida que valga la pena ser vivida" no es ajeno al debate sobre las necesidades básicas iniciado a principios de los años ochenta del siglo XX. Debate importante de recuperar porque obliga a reflexionar sobre las distintas dimensiones de la vida. Sin embargo, hay que advertir que dichos debates, mayoritariamente, también se han mantenido desde una mirada masculina, lo cual significa, que en la idea de necesidades básicas no se ha incorporado la experiencia histórica de las mujeres en el cuidado de las personas. Aspecto que, si no conociéramos la fuerza de las relaciones patriarcales, resultaría casi curioso: ¿cómo se pueden definir las necesidades básicas de las personas sin considerar la necesidad de cuidados, ya sean biológicos, afectivos u emocionales? Por tanto, la tarea vuelve necesariamente a ser la misma: ampliar la mirada en el concepto de necesidades básicas.

El desafío político se concreta en aceptar que es la sociedad en su conjunto la que debe asumir el cuidado de su población y no asignarlo a un sector de la población -las mujeres-, desplazando con ello hacia los hogares toda la tensión que conlleva la gestión del cuidado. Es la sociedad quien debe hacerse cargo de organizarlo de tal manera de dar respuesta a las necesidades humanas, sin que las personas que ejerzan de cuidadoras estén determinadas por alguna categoría social como clase, sexo o raza. Se plantea que la responsabilidad del cuidado no debería ser privada ni individual, sino social y política. Avanzar en esta línea significaría comenzar a revalorizar el trabajo de cuidados a la vez que reconocer la aportación a la sociedad y al bienestar de este tipo de trabajo, y así romper con la centralidad del trabajo mercantil como eje del funcionamiento social en términos generales y como generador de derechos sociales en términos más específicos.

En definitiva, integrar en el análisis económico toda la actividad desarrollada desde los hogares, no significa agregar a las mujeres al modelo vigente; representa algo mucho más profundo, una ruptura con lo establecido desplazando los objetivos desde el beneficio empresarial al cuidado de la vida humana. Ningún enfoque global social puede tener sentido si no tiene en cuenta las condiciones de reproducción de su población. Hacer explícito el trabajo doméstico y de cuidados en los esquemas económicos como trabajo necesario, no es sólo una cuestión de justicia, sino de sensatez y rigor si se pretende analizar e interpretar la realidad.

\section{Epílogo: crisis y condiciones de vida}

Es difícil acabar un escrito que pretende ofrecer una alternativa no sólo de análisis sino social y política y no relacionarlo con la crisis actual. De ahí este breve epílogo que no es sino cómo mirar la crisis desde la economía feminista.

Sabemos que la profunda crisis que estamos viviendo está afectando fundamentalmente a aquellos sectores más vulnerables de la sociedad, la mayoría de los trabajadores y trabajadoras que dependen directa o indirectamente de un salario 
para su subsistencia y los jóvenes de ambos sexos que no han podido incorporarse al empleo. Una crisis que está significando una redistribución salvaje de la renta y la riqueza y también de los tiempos y los trabajos. Una crisis que en definitiva está repercutiendo de forma brutal directamente en las condiciones de vida de las personas. Los efectos de la crisis están siendo durísimos para mucha gente, de distintos sexos y edades, y están causando enorme dolor. En particular, quisiera destacar los que están sufriendo las mujeres, como grupo social más vulnerable en una sociedad patriarcal.

Por una parte, la crisis está significando una reprivatización de la reproducción social. La drástica reducción del gasto público en servicios de cuidados, sanitarios o educativos, tiene como uno de sus fines -además de otros- que una parte de los cuidados regresen al hogar, con el consiguiente aumento del trabajo de las mujeres (recortes sanitarios, reducción de plazas de guardería, de plazas en residencias de personas mayores, de becas de comedor, etc.). Así, la reducción de gasto público se traspasa a los hogares en forma de incremento de trabajo. Pero el trabajo doméstico y de cuidados también se incrementa por la reducción de ingresos al hogar debido al paro, ya sea masculino o femenino: trabajos o producciones que se habían mercantilizado regresan al hogar, o, se deja de utilizar algún servicio por la imposibilidad de asumir los costes monetarios, aunque se asumen otros, como el incremento de trabajo (abuelas/os que se retiran de las residencias, criaturas que no van a guarderías, etc.). En definitiva, el trabajo doméstico y de cuidados está funcionando como amortiguador de los efectos devastadores de la crisis en el bienestar de las personas ${ }^{14}$.

Ahora bien, los problemas de falta de ingresos monetarios está aumentando las tensiones en las relaciones dentro de los hogares; tensiones, muchas de ellas, crea-

${ }^{14}$ Pero los hogares no son una caja negra y podríamos preguntarnos si el incremento de trabajo dentro de los hogares está repercutiendo por igual en mujeres y hombres. Las EET (hay que considerar que los recortes más fuertes son posteriores a la encuesta de 2009/2010) muestran que entre 2003 y 2010 los hombres han incrementado el trabajo doméstico y de cuidados en 24 minutos diarios como media social y las mujeres lo han reducido en 13 minutos, aunque las mujeres siguen realizando como media social 2 horas 13 minutos más que los hombres diariamente. En cambio, en relación al trabajo de mercado, ellos han reducido el tiempo de dedicación diario en 28 minutos (debido al desempleo) y ellas lo han incrementado en 13 minutos. Otra información de la encuesta hace pensar que los hombres que han incrementado el trabajo realizado en el hogar son fundamentalmente los desempleados: a) las edades donde los hombres han aumentado este tipo de trabajo son entre $30 \mathrm{y}$ 60 años, b) se ha reducido la doble presencia (realizar al menos diez horas semanales de cada trabajo) masculina en 14 puntos porcentuales y c) se ha incrementado en aproximadamente 14 puntos la dedicación masculina exclusivamente a trabajo de mercado (sin realizar al menos diez horas semanales de trabajo doméstico y de cuidados). Lo que es muy difícil de pronosticar es si este comportamiento de los hombres desempleados es transitorio mientras no cambie su situación laboral o si puede provocar un cambio de comportamiento permanente. 
das por la dificultad de la gestión de los cuidados; lo cual está incrementando todo tipo de violencia contra las mujeres en los hogares. Como indicador basta con observar el incremento del número de mujeres muertas por sus parejas o ex-parejas. Finalmente, y tal vez lo más grave es el retroceso político e ideológico que se produce en épocas de crisis. Este se produce en todos los campos, pero también afecta de forma específica a las mujeres. Como ejemplo a observar están las mujeres de América Latina -con diferencias importantes según países- que después de 25 años aún intentan recuperar espacios que estaban conquistados antes de la crisis de los años ochenta.

Pero todo ello no parece afectar -al menos no se percibe- a nuestros dirigentes políticos y económicos, preocupados como están por los problemas monetarios. Que los problemas monetarios estén relacionados con la vida real de las personas, es evidente; sin embargo, el tema es cómo se tratan, desde dónde se miran y cómo se enfocan según el objetivo social y político que se sostenga. Los enfoques habituales centrados en el mercado están incapacitados para ofrecer una visión global del problema que tenga como primer objetivo la vida de las personas. La ceguera de análisis, o más bien, el interés de un análisis sesgado y manipulado, impide ni siquiera vislumbrar una posible salida a la crisis.

Como se puso de manifiesto más arriba, desde hace ya tiempo, la economía feminista ha estado denunciando la escasa importancia que otorga la economía y la política a las condiciones de vida de las personas. Y es precisamente en ese punto que ha sido el objetivo central de este nuevo enfoque- donde la crisis se manifiesta de forma más dura. De ahí que un enfoque como el propuesto, que permite relacionar los distintos ámbitos sociales, económicos (y ecológicos) y tener en cuenta las interrelaciones y efectos entre todos ellos, con el único fin de conseguir mejores condiciones de vida para la población -mujeres y hombres-, se presenta como una alternativa analítica y política para enfrentar la actual situación. Como he manifestado en otro lugar, no se trata solo de una crisis financiera, sino de una crisis de reproducción social que es algo que va mucho más allá de una crisis financiera o económica. Y, por tanto, su significado obliga a examinar la naturaleza del sistema capitalista, su capacidad de reproducción y sostenibilidad y, particularmente, las tensiones profundas que aparecen en el terreno de la vida cotidiana, que afectan a personas "reales".

\section{Bibliografía}

Amoroso, M. I., et al. (2003). Malabaristas de la vida. Mujeres, tiempos y trabajos. Barcelona: Icaria editorial.

Balbo, L. (1980). Riparliamo del "welfare state: la società assistenciale, la società dei servizi. la società della crisi. Inchiesta. Núm. 46-47, [reproducido en 1982 en la revista $P A P E R S 18,51-85]$. 
Bosch, A., Carrasco, C y Grau E. (2005). Verde que te quiero violeta. Encuentros y desencuentros entre feminismo y ecologismo en Enric Tello, $L a$ historia cuenta (pp 321-346). Barcelona: El Viejo Topo.

Boulding, K. (1972). The Household as Achilles' Heel, Journal of Consumer Affairs. Vol. 6, Núm.1, 110-119.

- (1976). La economía del amor y del temor. Madrid: Alianza editorial (e.o.1973).

Carrasco, C. et al. (2006). Estadístiques sota sospita. Proposta de nous indicadors des de l'experiència femenina. Barcelona: Institut Català de les Dones.

Carrasco, C., Borderías, C. y Torns, T. (2011). El trabajo de cuidados: antecedentes históricos y debates actuales, en Cristina Carrasco, Cristina Borderías y Teresa Torns, El trabajo de cuidados. Historia, teoría y política (pp 13-95). Madrid: CIP-Ecosocial y La Catarata.

Federici, S. (2010a). Calibán y la bruja: Mujeres, cuerpos y acumulación originaria. Madrid: Traficantes de sueños. (e.o. 2004).

- (2010b). El trabajo precario desde un punto de vista feminista, www.sinpermiso.info

Folbre, N. (1995). Holding Hands at Midnight: The Paradox of Caring Labor. Feminist Economics Vol.1, Núm.1, 73-92.

Heather-Bigg, A. (1894). The Wife's Contribution to Family Income. The Economic Journal, Vol. 4, Núm. 13, 51-58.

Himmelweit, S. (1995). The Discovery of "Unpaid Work": The Social Consequences of the Expansion of "Work". Feminist Economics. Vol. 1, Núm. 2, 1-19.

Pateman, C. (1988). The sexual contract. Stanford: Stanford University Press. [Traducción castellana, El contrato sexual, Barcelona: Anthropos, 1995].

Pérez Orozco, A. (2006). Perspectivas feministas en torno a la economía: el caso de los cuidados. Madrid: Consejo Económico y Social, Colección Estudios, 190.

- (2007a). Amenaza tormenta: la crisis de los cuidados y la reorganización del sistema económico. Revista de Economía Crítica, Núm. 5, 7-37. (www.revistaeconomiacritica.org)

- (2007b). Global Care Chains. En Working Paper 2 Gender, Migration and Development Series, Santo Domingo: UN-INSTRAW.

Perkins Gilman, C. (1898). Women and Economics: A Study of the Economic Relation Between Men and Women as a Factor in Social Evolution. Boston: Small, 
Maynard \& Co. [Traducción castellana: Mujeres y economía: un estudio sobre la relación económica entre hombres y mujeres como factor de la evolución social. Biblioteca Javier Coy d'estudis nord-americans, Universitat de València, 2008]

Picchio, A. (2001). Un enfoque macroeconómico "ampliado" de las condiciones de vida, en Cristina Carrasco (coord.), Tiempos, trabajos y género (pp.15-37). Barcelona: Publicacions Universitat de Barcelona.

- (2009). Condiciones de vida: Perspectivas, análisis económico y políticas públicas. Revista de Economía Crítica, Núm. 7, 27-54. (www.revistaeconomiacritica.org).

Reid, M. (1934). Economics of Household Production, New York: John Wiley. 